\title{
A model of the late universe with viscous Zel'ldovich fluid and decaying vacuum
}

\author{
Rajagopalan Nair K and Titus K. Mathew, \\ Department of Physics, \\ Cochin University of Science and Technology, \\ Kochi-22, India.
}

\section{Introduction}

The recent cosmological observations have revealed that the present universe is accelerating in expansion [28, 33] due to the presence of an exotic component called dark energy which constitutes more than seventy percent of the total cosmic density. Earlier to this discovery, there were indications from weak [32] and strong[45] lensing phenomena, large scale structure [1], galaxy rotation [50, 34, 35], on the presence of another exotic component, the dark matter, which constitutes nearly $23 \%$ of the total density. The other known components are radiation and baryonic matter, comprising only about $1 \%$ and $5 \%$ respectively [32, 45, 11, 50, 34]. Till date there is no clear understanding regarding the nature of dark energy and dark matter. Many models have been proposed to explain the nature and evolution of these two hitherto unknown components. In the most successful model of the universe, the standard $\Lambda \mathrm{CDM}$ model, the dark matter is considered as some non-relativistic matter of zero pressure and dark energy is considered as cosmological constant. Due to the severe discrepancy between the observed and predicted values of the cosmological constant, called the cosmological constant problem, which doesn't have any natural explanation 
in the $\Lambda \mathrm{CDM}$ model, many alternative models have been considered in the recent literature with varying dark energy densities instead of pure cosmological constant [39]. Regarding dark matter also, there were no general consensus at all about its nature.

Even though the current data supports the exotic components like dark matter and dark energy, it is not really ruling out the existence of other exotic components. For instance, there exist speculations regarding the existence of a component called "dark radiation" [14. Another exotic component of interest is stiff fluid. In some early papers, Zel'dovich [47, 48] proposed a model in which the early stage of the universe is composed of stiff gas of baryonic particles with equation of state $p_{z}=\rho_{z}$, where $p_{z}$ is the pressure and $\rho_{z}$ is the density. This equation of state implies that the velocity of sound in such fluids will become equal to that of light[47]. Later many others studied the nature and evolution of such a stiff fluid in an expanding universe. Steili et al[43] have shown that the self interaction field between dark matter components will behave like stiff fluid and can be taken as an indication that the so called dark matter can be of stiff nature at least in the early stages of the universe. On investigating the early stages of the universe, Barrow [4] was able to establish the possible existence of Zel'dovich fluid (or stiff fluid). In dissipative cosmological models also, the presence of stiff fluid in the early stage of the universe has been speculated[13. The equation of state of the Zel'dovich fluid directly implies that the energy density of it decreases as, $\rho_{z} \propto a^{-6}$ as the universe expands, where $a$ is the scale factor of the universe. Because of this, the normal Zel'dovich fluid would have decoupled comparatively earlier to other components like radiation, which evolves as $a^{-4}$ and matter evolving as $a^{-3}$. Consequently the presence of normal Zel'dovich fluid would have a dominant effect only during the early stage of the universe. Inspired by these, the effect of stiff fluid on the primordial abundances of light elements was numerically computed by Dutta and Scherrer[15]. By comparing the prediction with observational abundance of light elements, especially with the abundance primordial helium-4, these authors have obtained a constraint on the Zel'dovich fluid density as, $\rho_{z} / \rho_{c}<30$, where $\rho_{c}$ is the critical density of the universe. Recently a simple cosmological model consisting of stiff fluid, non-relativistic matter and cosmological constant as dark energy was studied by Chavanis[12] and he has shown that, the universe began with a big-bang and will end in a de Sitter phase. 
Even though the conventional Zel'dovich fluid would not have any effect on the late stage of the universe, the Zel'dovich fluid with bulk viscosity could have strong influence on the later stage. Effect of bulk viscosity, especially on the late evolution of the universe was studied by many [8, 2, 23]. It is worthwhile to note the analayses of the bulk viscosity in the context of early inflation in reference[3]. A cosmological model with bulk viscous Zel'dovich fluid as the dominant cosmic component was analysed in reference[27]. This model predicts the late acceleration of the universe when the weighted bulk viscous coefficient is in the range $4<3 \zeta / H_{0}<6$. The authors have further studied the evolution of the model in the statefinder, $(r, s)$ plane, and have shown that the current state of the universe is arguably different from the standard $\Lambda$ CDM model. The statefinder parameters are the most suitable diagnostics to contrast the model with other relevant dark energy models, expecially $\Lambda \mathrm{CDM}$. The evolution of the parameters $r$ and $s$ and their present values are used to contrast the model. The $r-s$ plane is a two dimensional plane with $r$ and $s$ as the coordinates, used to show the comprehensive evolution of (these parameters) the model. For more details on statefinder analysis see [36]. The authors also proved that the model satisfies the generalized second law of thermodynamics at the Hubble horizon. This work was further extended in reference[31], where the authors have used the Union 2 supernovae data to constrain the possible value of the viscous coefficient and obtained $\zeta / H_{0} \sim 5.25$ for $H_{0} \sim 70 \mathrm{~km} / \mathrm{s} / \mathrm{Mpc}$, where $H_{0}$ is the Hubble parameter of the present epoch of the universe. However the age of the universe was found to be around 10 GY only. The over all behavior of this model is such that, it asymptotically evolves to a de Sitter one with deceleration parameter, $q \rightarrow-1$. The authors [31] have substantiated their results by performing a dynamical system analysis and have shown that the asymptotic de Sitter epoch is stable. Another important point, evident from their work is that the effective equation of state of the Zel'dovich fluid would start evolving from stiff nature $(p / \rho=1)$, pass through a state of pressureless matter $(p=0)$ and eventually tend towards, $p=-\rho$ corresponding to de Sitter epoch. So the inclusion of the bulk viscosity in the stiff matter would naturally take the fluid to manifest as pressureless matter and subsequently as a pure cosmological constant during the successive stages of the evolution of the universe. In spite of this reasonable evolution of the universe in the bulk viscous Zel'dovich 
fluid, the odd man out is the prediction regarding the age of the universe, which is less compared to the current observational results. In the present work we try to alleviate this drawback of the model by incorporating one more component, a varying vacuum energy as dark energy. We found that with such an addition in the cosmic components, the age of the universe can be enhanced to around $12 \mathrm{GY}$.

A scalar field model of the universe with both stiff fluid and an effective interacting vacuum were proposed by Cataldo et al.[11. The authors have considered a scalar field in FLRW universe and shown that, the field can effectively manifest as a mixture two barotropic fluids, one with an equation of state $p=\rho$, corresponding to the stiff fluid and the other with an equation of state $p=-\rho$ mimicking the vacuum. The self interaction of the field, which manifests as the interaction between the components, would allow the cosmological vacuum to become a dynamical quantity. We will further describes regarding this work in a later section.

In later works, many have found that there exists an alternate route to the decaying vacuum, which comes from quantum field theory techniques based on the renormalization group approach in curved spacetimes. The effective action in such theories inherits quantum effects from the matter sector. In general, the renormalization group techniques in curved spacetimes leads to a dependence of vacuum energy on the Hubble parameter $\mathrm{H}$ of the form, $\rho_{\Lambda}(H, \dot{H})=M_{P l}^{2} \Lambda(H, \dot{H})$ [40, 42, 38] and references therin).

In the present work we found that the addition of the decaying vacuum energy will not affect the asymptotic properties of the model, such that the end de Siter phase is still a stable one. The paper is organized as follows. In section 2, we discuss the scalar field representation of a model with a mixture of stiff fluid and interacting scalar field, which motivates the discussions in the following sections. In section 3 , we present the analytical solutions of the Hubble parameter, scale factor and the other cosmological parameters. In section 4 , we obtain the model paramters by constraining the model with supernovae observational data and then compute the evolution of the different cosmological parameters. We also evaluate the age of the universe in this section. Section 5 is devoted to the study of the asymptotic properties of this model followed by the conclusions in section 6 . 


\section{Scalar field approach to stiff fluid and interact- ing vacuum}

Let us consider the evolution of a self interacting scalar field, $\phi$. which is minimally coupled to gravity in flat isotropic and homogeneous universe. In describing the evolution of the scalar field we mainly follow the reference [11], so for more details see that reference. The evolution of the scalar field is governed by the equations

$$
3 H^{2}=8 \pi G \rho_{\phi}
$$

and

$$
\dot{\phi} \ddot{\phi}+3 H \dot{\phi}^{2}=\dot{\phi} \frac{d V(\phi)}{d \phi}
$$

where $H$ is the Hubble parameter, $V(\phi)$ is the potential, dot represents a derivative with respect to cosmic time and equation(2) represents the dynamical evolution of the field. The equation of state of the field is,

$$
\omega_{\phi}=\frac{p_{\phi}}{\rho_{\phi}}
$$

in which the pressure and density of the scalar field are given by,

$$
\rho_{\phi}=\frac{\dot{\phi}^{2}}{2}+V(\phi), \quad p_{\phi}=\frac{\dot{\phi}^{2}}{2}-V(\phi)
$$

The self interacting scalar field can be effectively treated as the mixture of two interacting fluids, with densities $\rho_{1}$ and $\rho_{2}$ which are having the equation of state $\omega_{1}$ and $\omega_{2}$ respectively. Then the effective pressure is,

$$
P_{e f f}=\omega_{e f f} \rho_{e f f}=\frac{\omega_{1} \rho_{1}+\omega_{2} \rho_{2}}{\rho_{1}+\rho_{2}} \rho_{e f f},
$$

where $\rho_{\text {eff }}=\rho_{1}+\rho_{2}$. Hence it is now possible to have two fluids, one with equation

of state, $\omega_{1}=1$, corresponding to stiff fluid and the other with equation of state, 
$\omega_{2}=-1$ corresponding to vacuum energy, if one identifies the corresponding densities and pressures as,

$$
\rho_{1}=\frac{\dot{\phi}^{2}}{2}, \quad p_{1}=\frac{\dot{\phi}^{2}}{2}
$$

and

$$
\rho_{2}=V(\phi), \quad p_{2}=-V(\phi) .
$$

One can either take these components as isolated from each other, so that each one of them satisfying separate conservation laws or can be taken as interacting components following the conservation equations,

$$
\dot{\rho}_{1}+3 H\left(\rho_{1}+p_{1}\right)=Q, \dot{\rho}_{2}+3 H\left(\rho_{2}+p_{2}\right)=-Q,
$$

for $Q>0$ the energy flows for $\rho_{2}$ to $\rho_{1}$ and for $Q<0$ the energy flow in the reverse direction. From equations (6), (7) and (8), it follows that,

$$
\ddot{\phi} \ddot{\phi}+3 H \dot{\phi}^{2}=Q(t), \quad \dot{\phi} \frac{d V(\phi)}{d \phi}=-Q .
$$

These equations are the equivalent evolution equation of the scalar field. Hence it is possible to consider a scalar field as a mixture of a stiff fluid interacting with an effective vacuum energy.

In reference [11], the authors have described the evolution of such a universe. Our aim is not in line with that. Instead we consider a phenomenological form for the decaying vacuum along with the bulk viscous stiff fluid (Zel'dovich fluid) and analyze both the background evolution of the universe, particularly in finding the age and also the asymptotic behavior.

\section{Background evolution with viscous Zel'dovich fluid and decaying vacuum $\Lambda(t)$}

We consider a flat FLRW universe with standard metric

$$
d s^{2}=-c^{2} d t^{2}+a^{2}(t)\left(d r^{2}+r^{2} d \theta^{2}+r^{2} \sin ^{2} \theta d \phi^{2}\right)
$$


where $t$ is the cosmic time, $(r, \theta, \phi)$ are the co-moving coordinates and $a(t)$ is the scale factor. The cosmic components are the bulk viscous Zel'dovich and a varying cosmological constant. A non-viscous ideal Zel'dovich fluid obeys the equation of state $p_{z}=\rho_{z}$ [47], which will change once the fluid is assumed to be viscous. Bulk viscosity can be arised due to the deviations of the system from local thermodynamic equilibrium[49]. It arises as an effective pressure to restore the system back to its equilibrium, whenever the cosmic fluid expands or contracts too fast. In incorporating the viscosity into the analysis we adopt the Eckart's formulation[17]. Landau and Lifshitz also discussed a formulation [26] equivalent to Eckart's. There had been works showing that the thermodynamical equillibria in Eckart's theory as unstable[18] and signals could propagate through the fluid at superluminal velocities[20]. But these difficulties can be overcome by taking account of the higher order terms. Such a more general formalism was developed by Israel et al [21, 22], to which Eckart's theory would appear as a first order limit. However owing to the simplicity many authors favor Eckart's formalism for a first step analysis. Later Hiscock and Salmonson [19] had shown that Eckart's formalism can be reasonably applied to FLRW universe with late acceleration.

For a first order deviation from thermodynamical equilibrium the energy momentum tensor can be written as,

$$
T^{\mu \nu}=\rho u^{\mu} u^{\nu}+(p+\Pi) h^{\mu \nu}
$$

where $\rho$ is the density of the fluid component, $u^{\mu}$ is the four velocity of an observer in Hubble flow, $(p+\Pi)$ is the effective pressure and $h^{\mu \nu}=u^{\mu} u^{\nu}+g^{\mu \nu}$, with $g^{\mu \nu}$ as the metric coefficients. The above mentioned approach results in the equation for effective pressure as $p^{\prime}=p+\Pi$ for the bulk viscous Zel'dovich fluid and it can be expressed as,

$$
p_{z}^{\prime}=p_{z}-3 \zeta H
$$

where we have $p=p_{z}$ the normal pressure, $\Pi=-3 \zeta H$, the viscous pressure and $H$ is the Hubble parameter. In cosmology, bulk viscosity arises as an effective pressure to restore the system back to its thermal equilibrium, which is broken when the cosmological fluid expands (or contracts) too fast. This bulk viscosity pressure generated ceases as soon as the fluid reaches the thermal equilibrium[46]. 
The second cosmic component in the present model is the time varying cosmological parameter given by [6],

$$
\Lambda(t)=3 \alpha H^{2}
$$

where $\alpha$ is a free parameter, value of which would be less than one. Earlier introduction of this kind of decaying vacuum was consdiered by Carvalho and Lima [9], where the authors restricted to $\alpha \leq 1 / 2$. A higher value of $\alpha$ resulted into incompatible age for the universe as claimed by many authors like [6], so the values of $\alpha$ is usually restricted to below one. Since this is effectively a form of time varying vacuum energy, its equation of state is taken as, $\omega_{\Lambda}=\left(p_{\Lambda} / \rho_{\Lambda}\right)=-1$. Basically the $\Lambda(t)$ models have been originated from curved space quantum field theories[41]. Often there appears a constant additive term along with the time varying part in the equation for $\Lambda(t)$, which, as argued by many [39, 29] facilitate the transition from the decelerating to an accelerating epoch of the expanding universe. But in the present model such an additive constant is not needed due to the presence of viscosity in the Zel'dovich fluid component, which will otherwise guarantee such a transition from an early deceleration to a later accelerating phase of expansion.

The Friedmann metric along with the standard Einstein's field equation will give the Friedmann equation for a flat universe as,

$$
3 H^{2}(t)=\rho_{z}+\rho_{\Lambda}
$$

where $\rho_{\Lambda}=\Lambda(t)$ (in standard units, $8 \pi G=1, c=1$ ), is the time varying cosmological parameter, equivalent to the standard dark energy density. These components together satisfy the conservation law (in the absence of any source, i.e. $Q=0$ ),

$$
\dot{\rho}_{\Lambda}+\dot{\rho}_{z}+3 H\left(\rho_{z}+p_{z}^{\prime}\right)=0 \text {. }
$$

where a dot represents a derivative with respect to cosmic time. Combining the Friedmann equation and the conservation equation, leads to

$$
\dot{\rho}_{z}+\dot{\rho}_{\Lambda}=-6 H\left(\rho_{z}-\frac{3}{2} \zeta H\right) .
$$


But from Friedmann equation, $\dot{\rho}_{z}+\dot{\rho}_{\Lambda}=6 H \dot{H}$. On substituting this, the above equation becomes,

$$
\dot{H}=-\left(\rho_{z}-\frac{3}{2} \zeta H\right) .
$$

Again from Friedmann equation one can substitute for $\rho_{z}$ as,

$$
\rho_{z}=3(1-\alpha) H^{2}
$$

From equations (17) and (18),

$$
\dot{H}+3 H\left((1-\alpha) H-\frac{\zeta}{2}\right)=0 .
$$

Solving equation (19) we obtain the Hubble parameter as,

$$
H(t)=\eta\left[1+\operatorname{coth}\left(3(1-\alpha) \eta\left(t-t_{0}\right)+\phi\right)\right]
$$

where $\eta=\frac{\zeta}{4(1-\alpha)}, \phi=\operatorname{coth}^{-1}\left(\frac{H_{0}}{\eta}-1\right)$ and $H_{0}$ is the current value of Hubble parameter. Integrating the above equation, we obtain the equation for the scale factor as

$$
a(t)=e^{\eta\left(t-t_{0}\right)}\left(\frac{\sinh \left[3 \eta(1-\alpha)\left(t-t_{0}\right)+\phi\right]}{\sinh (\phi)}\right)^{\frac{1}{3(1-\alpha)}} .
$$

Using this equation, the Hubble parameter in equation (20) can be recast as,

$$
H=\frac{\zeta}{2(1-\alpha)}+\left(H_{0}-\frac{\zeta}{2(1-\alpha)}\right) a^{-3(1-\alpha)} .
$$

In the asymptotic limit $a(t) \rightarrow \infty$ the Hubble parameter becomes a constant, $H \rightarrow$ $\frac{\zeta}{2(1-\alpha)}$ which corresponds to the de Sitter phase with exponential increase in the scale factor, while in the limit $a(t) \rightarrow 0$, the Hubble parameter evolves as, $H \sim$ $a^{-3(1-\alpha)}$, which points to an earlier decelerated epoch dominated with Zel'dovich fluid with density $\rho_{z} \sim H^{2} \sim a^{-6(1-\alpha)}$. Thus the existence of the transition from an early 
decelerated to a late accelerated epoch is guaranteed. In these limits the scale factor will evolve as follows. As $t \rightarrow \infty$ the scale factor will evolve as, $a(t) \rightarrow e^{2 \eta\left(t-t_{0}\right)}$, this exponential increase corresponds to the de Sitter epoch, while in the early stage of the evolution, corresponding to $3 \eta(1-\alpha)\left(t-t_{0}\right)<1$ the above form of $a(t)$ almost implies that, $a(t) \sim\left(\left(1+3 \eta(1-\alpha)\left(t-t_{0}\right)\right)^{1 / 3(1-\alpha)}\right.$, representing a decelerating epoch. What is important here is that the transition occurs without the aid of an additive constant in the $\Lambda(t)$. In non-viscous models like entropic dark energy [5] or Ricci dark energy [29], the presence of a bare constant cosmological term is essential for having a transition from the early decelerated epoch to the late accelerated epoch.

The evolution of the cosmological parameters, like Hubble parameter, scale factor etc are depending upon the numerical values of the model parameters $\alpha$ and viscous coefficient $\zeta$. However it is clear from the expression of scale factor in equation(21) that for a constant $\alpha$ the beginning of the universe corresponding to $a=0$ would have occurred earlier into the past of the universe as $\zeta$ assumes higher values. For constant $\zeta$ and increasing $\alpha$, the situation will be the same too. In both the cases the age of the universe increases compared to a model with only Zel'dovich fluid as the cosmic component. However, only with an extraction of these parameters, a final conclusion regarding the age of the universe can be made.

We have considered a flat universe $(k=0)$, since observations strongly indicate that our universe is flat [25]. The inflationary models theoretically propose a very small value for curvature around $\Omega_{k 0} \sim 10^{-5}$ while observations favor a value of the order of $10^{-2}$. Basically for non-flat universe, the Friedmann equation becomes,

$$
3 H^{2}=\rho_{z}+\rho_{\Lambda}+\rho_{k} .
$$

where $\rho_{k}=-k a^{-2}$. Since the interaction is only between Zel'dovizh fluid and the vacuum, the conservation law is,

$$
\dot{\rho}_{z}+\dot{\rho}_{\Lambda}+3 H\left(\rho_{z}+p_{z}^{\prime}\right)=0 .
$$

Using equations $(12)$ and $(18)$ and through simple algebra we can rewrite the above equation as,

$$
\dot{H}+3 H\left((1-\alpha) H-\frac{\zeta}{2}\right)=\dot{\rho}_{k} .
$$


In our original work we took, $\rho_{k}=0$ consequently the rhs of the above equation is zero. But for non-flat universe the contribution due to the rhs term, $\dot{\rho}_{k}=-2 H \rho_{k}$ is extremly small especially in the late stage, first of all due to the decreasing nature of $H$ and secondly due to the extremely low magnitude of $\rho_{k}$. Hence the solution of the above equation (our model) would be almost close to the solution of the corresponding homogeneous equation with zero curvature.

\section{Extraction of model parameters and evolution of cosmic parameters}

The best fit values for $\zeta, \alpha$ and $H_{0}$ are estimated using type Ia supernova observational data. Union data containing 307 data points [24] in the red shift range $0.01<z<1.55$ has been used here. For $i^{\text {th }}$ supernova at a red shift $z$, having an apparent magnitude $m$ and absolute magnitude $M$, the distance modulus is,

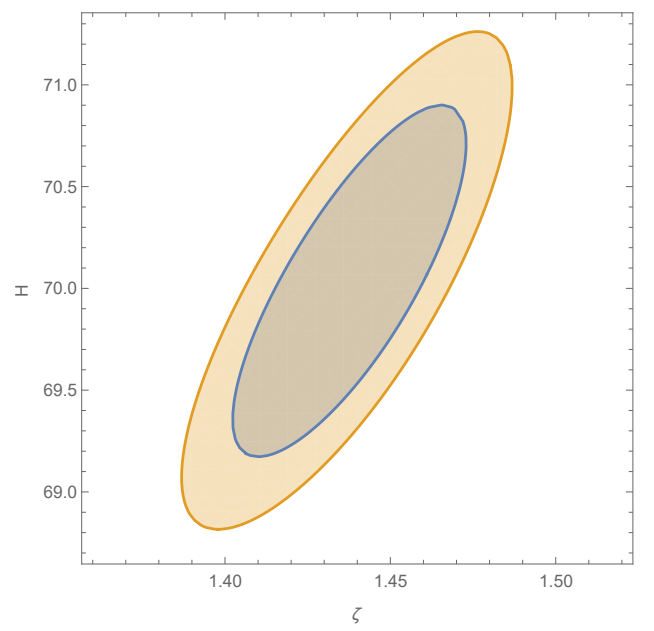

Figure 1: Contour plot for the parameters $H$ and $\bar{\zeta}$ for fixed $\alpha=0.14$.

$$
\mu_{i}(z)=(m-M)=5 \log _{10} d_{L}(z)+25,
$$


where $d_{L}(z)$ is its luminosity distance, which is depending on the model parameters also and is given by

$$
d_{L}(z, \bar{\zeta}, \alpha)=\frac{c(1+z)}{H_{0}} \int_{0}^{z} \frac{d z^{\prime}}{h\left(z^{\prime}, \bar{\zeta}, \alpha\right)}
$$

where $h\left(z^{\prime}, \bar{\zeta}, \alpha\right)=\frac{H\left(z^{\prime}, \bar{\zeta}, \alpha\right)}{H_{0}}$. Here we have redefined the viscosity coefficient by weighting it with the present Hubble parameter as, $\bar{\zeta}=\frac{\zeta}{H_{0}}$. Equation for $h$ in terms of $z$, the cosmological red shift is obtained by substituting for scale factor using $a=\frac{1}{1+z}$ in equation 22 as

$$
h\left(z^{\prime}, \bar{\zeta}, \alpha\right)=\frac{\bar{\zeta}}{2(1-\alpha)}+\left(1-\frac{\bar{\zeta}}{2(1-\alpha)}\right)(1+z)^{3(1-\alpha)}
$$

The theoretical distance moduli for various red shifts are obtained using equation (27) and are compared with the corresponding observational data. The statistical $\chi^{2}$ function for comparing the theoretical and observational values of the distance moduli is defined as,

$$
\chi^{2}=\sum_{i=1}^{n} \frac{\left[\mu_{i t h}-\mu_{i o b}\right]^{2}}{\sigma_{i}^{2}}
$$

where $\mu_{i t h}$ is the theoretical value of the distance modulus of the $i^{\text {th }}$ supernova for a given redshift and $\mu_{i o b}$ is its observed distance modulus corresponding to the same redshift. $\sigma_{i}$ is the variance of the measurement of $i^{t h}$ supernova and $n=307$ is the number of data points. $\mu_{i t h}$ being dependent on $\left(\alpha, \bar{\zeta}, H_{0}\right)$, the best estimates of the parameters $\left(\bar{\zeta}, \alpha, H_{0}\right)$ are obtained by minimizing the $\chi^{2}$ function. The minimum of the $\chi^{2}$ indicates the goodness-of-fit of the model apart from giving the best estimates of the model parameters.

For obtaining the $\chi^{2}$ function we also used Background (CMB) data from the WMAP 7-yr observation and the Baryon Acoustic Oscillation (BAO) data from Sloan Digital Sky Survey(SDSS). The BAO signal has been directly detected by SDSS 
survey at a scale $\sim 100 \mathrm{MPc}$. The BAO peak parameter value was first proposed by Eisenstein, D. J. et al[16] and is defined as

$$
\mathcal{A}=\frac{\sqrt{\Omega_{m}}}{h\left(z_{1}\right)^{\frac{1}{3}}}\left(\frac{1}{z_{1}} \int_{0}^{z_{1}} \frac{d z}{h(z)}\right)^{\frac{2}{3}}
$$

Here $\mathrm{h}(\mathrm{z})$ is the Hubble parameter, $z_{1}=0.35$ is the red shift of the SDSS sample. Using SDSS data from luminous red galaxies survey the value of the parameter $\mathcal{A}$ (for flat universe) is given by $\mathcal{A}=0.469 \pm 0.017[16]$. The $\chi^{2}$ function for the BAO measurement takes the form

$$
\chi_{B A O}^{2}=\frac{(\mathcal{A}-0.469)^{2}}{(0.017)^{2}}
$$

The CMB shift parameter is the first peak of CMB power spectrum[7] which can be written as

$$
\mathcal{R}=\sqrt{\Omega_{m}} \int_{0}^{z_{2}} \frac{d z}{h(z)}
$$

Here $z_{2}$ is the red shift at the last scattering surface. From the WMAP 7-year data, $z_{2}=1091.3$. At this red shift $z_{2}$, the value of shift parameter would be $\mathcal{R}=1.725 \pm$ 0.018 [25]. The $\chi^{2}$ function for the CMB measurement can be written as

$$
\chi_{C M B}^{2}=\frac{(\mathcal{R}-1.725)^{2}}{(0.018)^{2}}
$$

Considering three cosmological data sets together, i.e. $(\mathrm{SNe}+\mathrm{BAO}+\mathrm{CMB})$, the total $\chi^{2}$ function is then given by

$$
\chi_{\text {total }}^{2}=\chi_{S N e}^{2}+\chi_{B A O}^{2}+\chi_{C M B}^{2}
$$

By minimising the $\chi^{2}$, we found parameter values as, $\alpha=0.14, H_{0}=70.3 \mathrm{~km} / \mathrm{s} / \mathrm{Mpc}$, and $\bar{\zeta}=$ 1.446 for $\chi^{2}$ per degrees of freedom, $\chi_{d o f}^{2}=\frac{\chi_{\min }^{2}}{n-m}=1.016$, where $n$ is the number of data points and $m=3$ the number of free parameters. We have constructed the confidence interval plane for the parameters $H$ and $\bar{\zeta}$ keeping $\alpha=0.14$, its best estimated 
value. The confidence intervals corresponding to $68.4 \%$ and $95.4 \%$ show fairly good behavior and are given in figure.1. The best fit values for the parameters $H_{0}$ and $\bar{\zeta}$ with corrections for a confidence of $64.8 \%$ are $H_{0}=70.03_{-0.46}^{+0.54}$ and $\bar{\zeta}=1.446_{-0.023}^{+0.018}$. For $95.4 \%$ probability the corrected parameter values are $H_{0}=70.3_{-0.47}^{+0.565}$ and $\bar{\zeta}=$ $1,446_{0.032}^{+0.095}$ for $\alpha=0.14$. It may be noted that in reference [6], the authors have extracted an upper limit for the parameter $\alpha$ by constraining a model with a decaying vacuum, $\Lambda=\Lambda_{0}+3 \alpha H^{2}$, as $\alpha \leq 0.15$.

In discussing the evolution of different cosmological parameters, it is better to start with the equation of state parameter. As it was shown in some of the earlier works [27], the equation of state of the viscous Zel'dovich fluid has natural evolution from its extreme stiff nature (corresponds $\omega=1$ ) to the de Sitter type behavior through radiation (corresponds to $\omega=1 / 3$ ) like and matter like (corresponds to $\omega=0$ ) natures. First we will consider the net equation of state, comprising both the Zel'dovich fluid and the decaying vacuum, which can be obtained by the standard procedure as,

$$
\omega(z)=-1-\frac{1}{3} \frac{d}{d x}\left(\ln h^{2}\right)
$$

From the equations (28) and (35), the equation of state can be expressed as,

$$
\omega(z)=-1+(2(1-\alpha)-\bar{\zeta}) \frac{1}{h}(1+z)^{3(1-\alpha)} .
$$

For $\alpha=0$, and $\bar{\zeta}=0$ equation of state tends to $\omega(z) \rightarrow 1$ for very large redshift, which corresponds to the early epoch dominated with Zel'dovich fluid with negligible viscosity. For the chosen value of the parameter, $\alpha=0.14$ a transition into the late accelerating phase would occur for a range of values of the viscous coefficient, $1.72>\bar{\zeta}>0$. For the best fit values of the parameters, $\left(\alpha, \bar{\zeta}, H_{0}\right)=(0.14,1.445,70.03)$ the evolution of the equation of state parameter is as expected (see figure 2), that is, starting with a value corresponding to stiff fluid and gradually approaching the value corresponding to de Sitter epoch. The current value of $\omega$ corresponding to the best fit values of the parameters is found to be $\omega \sim-0.72$, very much close to the range of values of the equation of state from WMAP data[25]. 


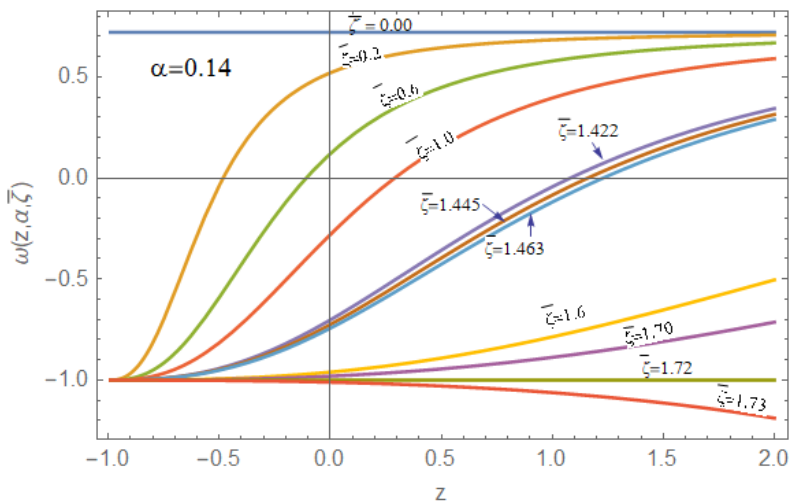

Figure 2: Evolution of $\omega$ with time for constant $\alpha=0.14$ and varied values of the viscous coefficient, $\bar{\zeta}$.

The equation of state of the viscous Zel'dovich fluid can be obtained using a similar approach as,

$$
\omega_{z}=-1-\frac{1}{3} \frac{d \Omega_{z}}{d x}=-1-\frac{(1-\alpha)}{3} \frac{d \ln h^{2}}{d x} .
$$

The difference in $\omega_{z}$ compared to $\omega(z)$ is that the second term on the right hand side of the above equation contains an extra term, $(1-\alpha)$, so that the final expression for $\omega_{z}$ becomes,

$$
\omega_{z}=-1+(1-\alpha)(2(1-\alpha)-\bar{\zeta}) \frac{1}{h}(1+z)^{3(1-\alpha)} .
$$

The general evolution of $\omega_{z}$ is hence similar to that of $\omega(z)$ except in the particular numerical values corresponding to different epochs. But both will approach the de Sitter value as $a \rightarrow \infty$.

The deceleration parameter $q(z)$ is a measure of the acceleration and can be obtained from the basic equation,

$$
q=-1-\frac{\dot{H}}{H^{2}}
$$

is also evaluated. Using equation(22), deceleration parameter takes the form 


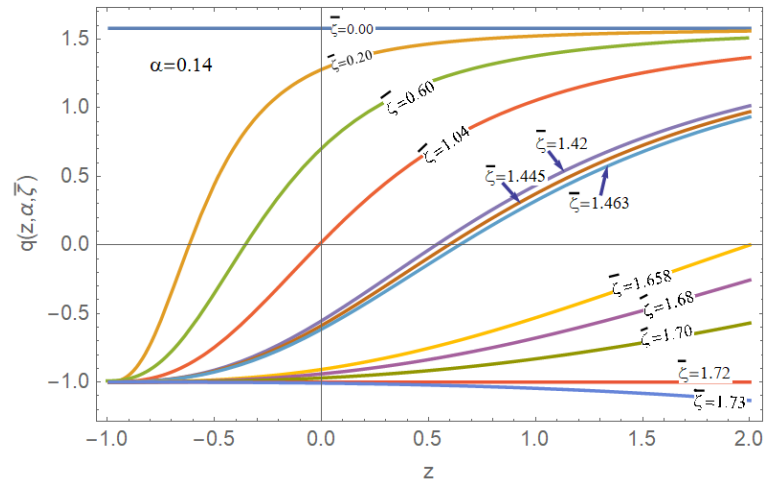

Figure 3: Evolution of deceleration parameter with $\alpha=0.14$ and varying $\bar{\zeta}$.

$$
q=-1+\left(\frac{3(1-\alpha)}{1+\left(\frac{1}{\frac{2(1-\alpha)}{\widetilde{\zeta}}-1}\right)(1+z)^{-3(1-\alpha)}}\right)
$$

When both the model parameters $\alpha$ and $\bar{\zeta}$ are equal to zero, the cosmic component becomes pure Zel'dovich fluid and $q=2$. For the best estimated values of the model parameters, the transition is found to occur at a redshift, $z \approx 0.61$ which is again close to the observed value. Irrespective of the values of $\bar{\zeta}$ the deceleration parameter asymptotically approaches the value, $q=-1$.

Finally we will discuss about the evolution of the scale factor. The age of the universe can be directly obtained for the evolution of the scale factor. The evolution of the scale factor is given in equation(21). We have already shown its asymptotic behavior in a previous section, that in the early epoch it evolves as in the decelerated phase and in the extreme future it evolves as in de Sitter epoch. In general, the form of $a(t)$ indicates the presence of big-bang as $\left(t-t_{0}\right) \rightarrow-\infty$. The evolution of it as shown in Figure (4) for the best fit values of the model parameters indicate it. But for higher values of $\zeta$ it is found that the big-bang occur at earlier times as evident from figure (5). It is also clear that, for extremely high values of the viscous parameter, the scale factor would have no-zero values in the beginning indicating the absence of big-bang. It is found that, there is no big-bang in the model for $\bar{\zeta}>1.72$. This means 


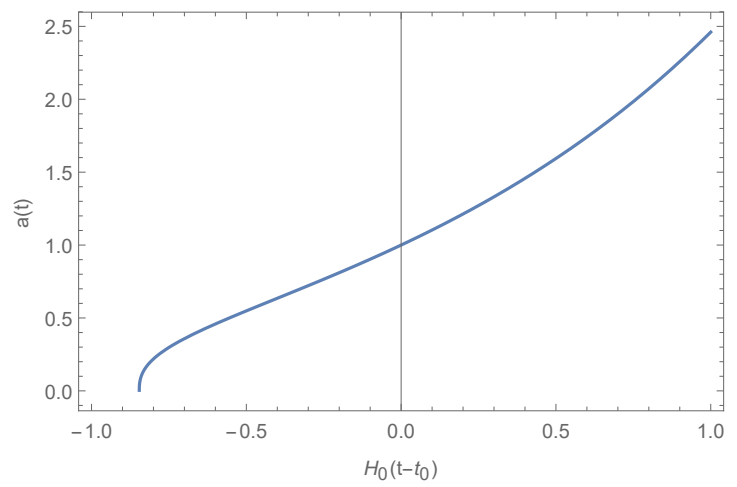

Figure 4: Evolution of scale factor with time. The profile corresponds to best fit values, $\alpha=0.14, \bar{\zeta}=1.445$ and $H_{0}=70.03 \mathrm{~km}^{-1} \mathrm{~s}^{-1} \mathrm{Mpc} \mathrm{c}^{-1}$.

that age of the universe defined only for $\bar{\zeta}<1.72$.

The age of the universe in the present model can be obtained by equating the scale factor to zero, which leads to,

$$
S h\left[3 \eta(1-\alpha)\left(t_{B}-t_{0}\right)+\phi\right]=0 .
$$

where we took, $t=t_{B}$, as the big bang time. This leads to the equation of the age of the universe as,

$$
t_{0}-t_{B}=\frac{\phi}{3 \eta(1-\alpha)} .
$$

On substituting the expressions for $\phi$ and $\eta$, the above equation can be simplified into,

$$
t_{0}-t_{B}=\frac{2}{3 \bar{\zeta}} \ln \left(\frac{1}{1-2 \bar{\eta}}\right) H_{0}^{-1},
$$

where $\bar{\eta}=\eta / H_{0}$. For the best estimated values of the model parameters, it is found that, the above equation gives an age of the universe in the range, $11.39-12.18 \mathrm{GY}$. First of all, this age is higher than the age predicted by the model in which Zel'dovich fluid is the dominant component[31]. Secondly it is near to the age of the universe obtained from the data on oldest globular clusters[30, 10, 37, 44]. In this sense the 


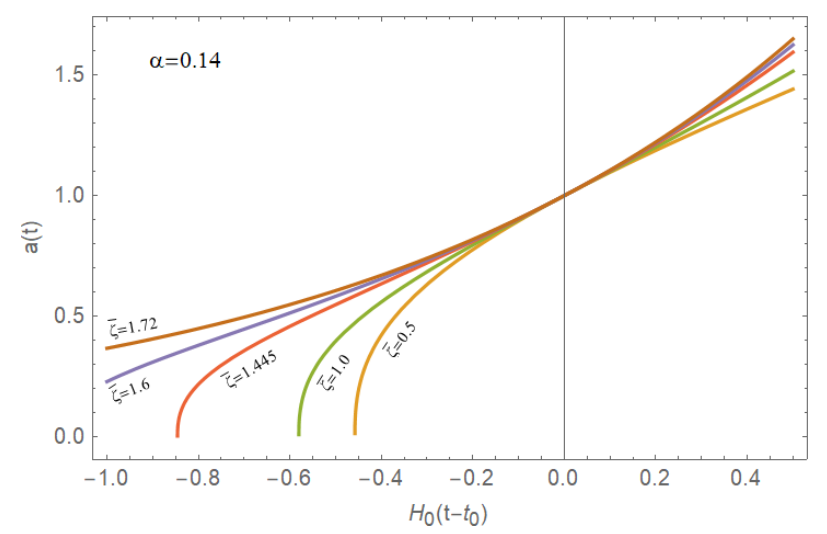

Figure 5: The spectrum of curves on evolution of $a(t)$ with $\alpha=0.14$ and varying $\bar{\zeta}$. There is big-bang for $\bar{\zeta}<1.72$.

model seems to solve the problem of age which existed in the model with Zel'dovich fluid as the only cosmic component. So it can be concluded that, the inclusion of a varying dark energy component along the bulk viscous Zel'dovich fluid is essential for the consistency with the age determination of the present universe.

The analysis so far reveals that the inclusion of the additional component, the decaying vacuum to the viscous Zel'dovich fluid, the model gives a reasonable back ground evolution of the universe. Apart from this, the age of the resulting universe will be high compared to the model with Zel'dovich fluid alone as the cosmic component. The viscous Zel'dovich fluid component evolves in such a way that, during the very early period the matter component is a stiff fluid, compatible with many theoretical speculations [15]. But as the universe evolves, the equation of state is smoothly evolving towards that of pressureless fluid, corresponding to the non-relativistic matter. Hence the model supporting the speculation that in the early period the matter would have existed as a stiff fluid. In the late stage, the evolution is compatible with the standard $\Lambda \mathrm{CDM}$ model, in predicting the observational parameters, including the age of the universe. In next section we do a dynamical system analysis of the model, which may throw more light on the viability of the present model. 


\section{Dynamical system analysis}

Dynamical system analysis is an effective method to extract the useful information about the stability of the asymptotic behavior of the model. For this, we have to express the cosmological equations governing the evolution of the model as a set of autonomous differential equations. Then the concerned information can be obtained by finding critical points and analyzing the nature of the trajectories in the neigh-

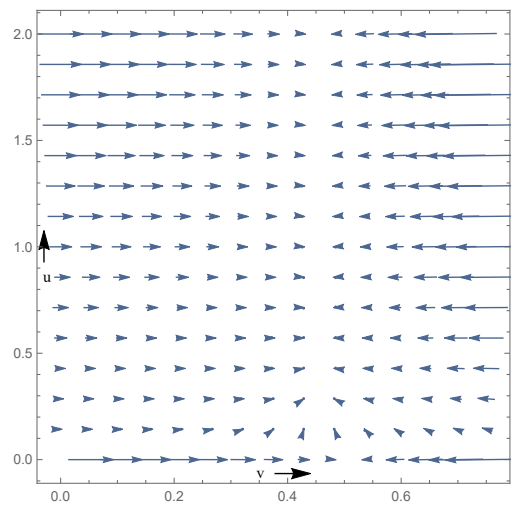

Figure 6: Plot of vector field of the phase space around the critical point $(0.607,0.457)$. The arrowhead of trajectories are tilted towards the critical point.

borhood of the critical points. Eventually it will become clear from this analysis whether the model in question is consistent with the presently accepted cosmological paradigm. In the present case this means whether the model predicts a stable evolution from a pre-decelerated epoch to a later accelerated epoch.

The first step in the dynamical system analysis is to identify proper phase-space variables. In the present case, we define,

$$
u=\Omega_{z}=(1-\alpha) h^{2}, \quad v=\frac{1}{1+\frac{1}{h}}
$$

as the phase-space variables where the quantities have their usual meaning. These variables will take the range, $0 \leq u \leq 1$ and $0 \leq v \leq 1$. The resulting coupled au- 
tonomous differential equations can then be formed using the Friemdmann equations and they are,

$$
\dot{u}=6 H_{0} \frac{u}{1-\alpha}\left(\frac{\bar{\zeta}}{2}-\frac{(1-\alpha) v}{1-v}\right)
$$

and

$$
\frac{\dot{v}}{\sqrt{1-\alpha}}=3 H_{0} v^{2}\left(\frac{\bar{\zeta}}{2 \sqrt{u}}-\sqrt{1-\alpha}\right) .
$$

The points in the phase space exhibit isomorphism with the exact solutions of cos-

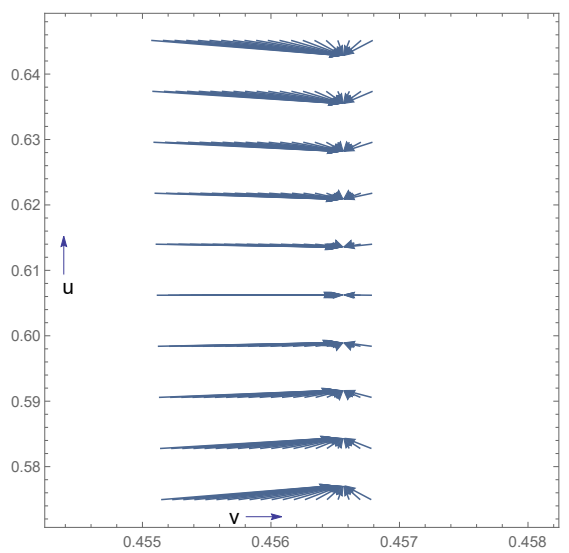

Figure 7: Plot of vector field of the phase space close to the critical point.

mological field equations. The ODEs are easier to be solved when the derivatives in them are written in terms of $\tau=\ln (a)$. The behavior of ODEs in the linear, closeby regions of critical points can be expressed in terms of a matrix equation accommodating $u^{\prime}=\frac{d u}{d \tau}$ and $v^{\prime}=\frac{d v}{d \tau}$ enabling flux analysis in terms of $\tau$ parameter of the equivalent autonomous ODE system. We then establish the correspondence of the dynamics of $\rho_{z}=\rho_{z}(t), \rho_{\Lambda}=\rho_{\Lambda}(t)$ and $H=H(t)$ with the mentioned simplified flux analysis in phase space for small perturbations in the linear limit $u \rightarrow u+\delta u(\tau)$ and $v \rightarrow v+\delta v(\tau)$, where $\delta u$ and $\delta v$ are the perturbations. The critical points are the solutions of the algebraic equations $P(u, v)=0$ and $Q(u, v)=0$ where 


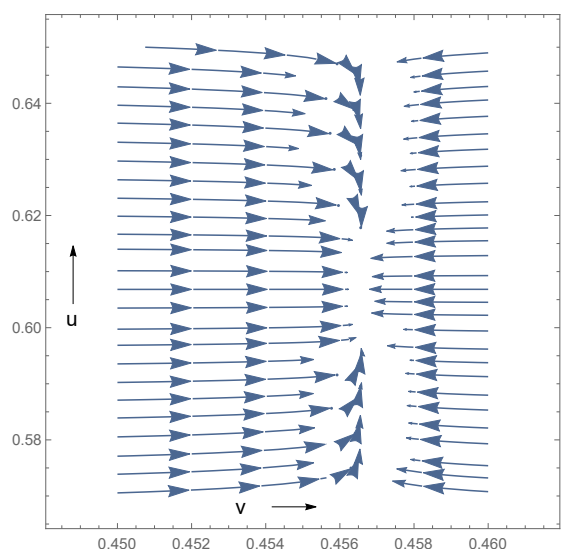

Figure 8: Stream plot of the trajectories around the critical point $(0.607,0.457)$

$P(u, v)=6 H_{0} \frac{u}{1-\alpha}\left(\frac{\bar{\zeta}}{2}-\frac{(1-\alpha) v}{1-v}\right)$ and $Q(u, v)=3 H_{0} v^{2}\left(\frac{\bar{\zeta}}{2 \sqrt{u}}-\sqrt{1-\alpha}\right)$. The perturbations around the critical points satisfy the equation,

$$
\left(\begin{array}{c}
\delta u^{\prime} \\
\delta v^{\prime}
\end{array}\right)=\left(\begin{array}{cc}
\left(\frac{\partial P}{\partial u}\right)_{0} & \left(\frac{\partial P}{\partial v}\right)_{0} \\
\left(\frac{\partial Q}{\partial u}\right)_{0} & \left(\frac{\partial Q}{\partial v}\right)_{0}
\end{array}\right)\left(\begin{array}{c}
\delta u \\
\delta v
\end{array}\right)
$$

where the suffix, ${ }^{\prime} 0^{\prime}$ denotes the value at the critical point and $2 \times 2$ matrix in the above equation is the Jacobian. The nature of the eigen values of the Jacobian matrix determine the behavior of the system near the critical points.

The critical point of interest corresponding to the equations (45) and (46) is $\left(u_{c}, v_{c}\right)=(0.607,0.457)$. It can be seen that the critical value $v_{c}$ corresponds to the end de Sitter epoch, where the Hubble parameter becomes, $h \rightarrow \frac{\bar{\zeta}}{2(1-\alpha)}$. Then using the relation $v=\frac{1}{1+\frac{1}{h}}$ and the best fit values of the model parameter, it can be seen that $v_{c}=0.457$ for the end de Sitter phase. The value $u_{c}=0.607$, the mass parameter of the bulk viscous Zel'dovich fluid also corresponds to the end de Sitter epoch. That is, in the end de Sitter epoch, $u \rightarrow \frac{\bar{\zeta}^{2}}{4(1-\alpha)}$, which for the best estimated values of the model parameters will become equal to 0.607 and is $u_{c}$. The eigen values corresponding to this critical point is $(0,-4.11)$. 
The first eigenvalue, 0 apparently suggests the absence of any isolated critical point. But it is seen that such a situation does not arise originally from the ODEs by setting $\dot{u}=0$ and $\dot{v}=0$. So the apparent discrepancy suggestive of lack of an isolated critical point arises from the errors of linear approximation of trajectory flux of the system in its immediate neighborhood. A low resolution view of vector field in phasespace is depicted in the figure (6). Higher resolution vector field plot as in figure (7) makes the view of the critical point as an attractor. The high resolution stream plot as in figure(8) also is a clear indicator that the critical point is an attractor.

\section{Conclusion}

Many have speculated that matter present in the early stage of the universe were of stiff nature, with equation of state, $p / \rho=1$. But owing to the fast decrease in its energy density, it would have effect only on processes like primordial nucleosynthesis. Later works which studied the bulk viscous stiff fluid, found that they can even cause the late acceleration of the universe. But the main drawback of such models were that, they predicted less age for the universe. In the present work we have studied a model with Bulk viscous stiff fluid and decaying vacuum energy as cosmic components. We found that the model possesses reasonably good back ground evolution, so as to produce a late acceleration at about a redshift compatible with the observational results. The model also predicts a de Sitter epoch as the end phase. It is found that the acceleration is mainly due to the effect of bulk viscosity, because for the decaying vacuum to produce a transition into the late acceleration, there has to be a constant in the vacuum energy density. But the decaying vacuum we have considered doesn't have such a constant. The effect of varying vacuum energy is reflected in the age of the universe. It was found that the age of the universe was increased compared to the model with viscous Zel'dovich fluid alone as the component. Age obtained is in agreement with the age deduced from the observations of the oldest globular clusters.

It was found that the equation of state of the fluid starts form the stiff nature, but eventually reduces to that of the matter and finally goes over to that of a pure cosmological constant corresponding to the de Sitter epoch. The dynamical system analysis shows that, the end de Sitter phase is a stable one. During this stable end de 
Sitter phase, the density of the Zel'dovich fluid is found to be around 0.6, which itself confirms that the late phase of the universe in this model is dominantly controlled by the viscous nature of the Zel'dovich fluid rather than the decaying vacuum energy. Acknowledgement One of the authors (KRN) thanks CUSAT Kochi, India for financial support in the form of a research fellowship. We also wish to thank IUCAA, Pune, India for the local hospitality during our visits, where part of the work had been carried out.

\section{References}

[1] Allen, S. W., Fabian, A. C., Schmidt, R. W. and Ebeling, H., 2003, Mon. Not. Roy. Astron. Soc., 342, 287.

[2] Athira Sasidharan and Titus K. Mathew, 2015, Eur. Phys. J. C, 75, 348.

[3] Bamba, K. and Odintsov, S. D., 2016, Eur. Phys. J C 7618.

[4] Barrow, J. D., 1986, Phys. Lett. B, 180, 335.

[5] Basilokos, S. and Sola, J., 2014, Phys. Rev. D, 90, 023008.

[6] Bessada, D. and Miranda, O. D., 2013, Phys. Rev. D, 88, 08353.

[7] Bond, J. R. et al, 1997, Mon. Not. Roy. Astron. Soc. 291 L33

[8] Brevik, I., Gron, O., De Haro, J., Odintsov, S. D. and Saridakis, E. N., 2017, Int. J. Mod. Phys. D, 26, 1730024.

[9] Carvalho J. C. and Lima J. A. S., 1992, Phys. Rev. D 462404

[10] Carretta, E., Gratton, R. G., Clementini, G. and Fucipecci, F., 2000, Astrophys.J., 533, 215.

[11] Cataldo, M., Arevalo, F. and Mella, P., 2013, Astrophys. Sapce Sci., 344, 495 
[12] Chavanis, P., 2015, Phys. Rev. D, 92, 103004.

[13] Cruz, M., Cruz, N. and Leppe, S., 2017, Phys. Rev. D, 96, 124020

[14] Dutta, S., Hsu, S. D. H., Reeba, D. and Sherrer, R. J., 2009, Phys. Rev. D, 79, 103504 .

[15] S. Dutta and R. J. Scherrer, 2010, Phys. Rev. D, 82, 083501.

[16] Eisenstein, D. J. et al. [SDSS Collaboration], 2005 Astrophys. J. 633560.

[17] Eckart, E., 1940, Phys. Rev., 58, 919.

[18] Hiscock, W. A., and Lindblom, L., 1985, Phys. Rev. D, 31, 725.

[19] Hiscock, W. A. and Salmonson, J., 1991, Phys.Rev. D, 43, 3249.

[20] Israel, W., 1976, Ann. Phys.(NY)100 310.

[21] Israel, W., and Stewart, J. M., 1979, Ann. Phys.(NY), 118, 341.

[22] Israel, W and Stewart, J. M., 1979, Proc. R. Soc. Lond. A, 365, 43.

[23] Jerin Mohan N. D., Athira Sasidharan and Titus K. Mathew, 2017 Eur. Phys. J. C, 77849

[24] Kovalsky, M., et al., 2008, Astrophys. J., 686, 749.

[25] Komatsu, E., et al., WMAP Collaboration, 2011, Astro. Phys. J. Suppl., 192, 18.

[26] Landau, L. D., and Lifshitz, E. M., Fluid Mechanics (Adsiison-Wesley,(1958.)

[27] Mathew, T. K., Aswati, M. B. and Manoj, M., 2014, Eur. Phys. J. C, 74, 3188.

[28] Perlmutter, S., et al., 1999, Astrophys. J., 517, 565.

[29] Paxy, G. and Titus K. Mathew, 2016, Mod. Phys. Lett. A, 31, 1650075. 
[30] Pont, F., Mayor, M., Turon, C. and Vandenberg, D. A., 1988, A.\&A., 329, 87.

[31] Rajagopalan Nair, K. and Titus K. Mathew, 2016, Eur. Phys. J. C, 76, 519.

[32] Refregier, A., 2003, Ann. Rev. Astron. Astrophys., 41, 645.

[33] Riess, A. G., et al., 1988, Astron. J., 116, 1009.

[34] Rubin, V. C. and Ford, W. K. J., 1970, Astrophys. J., 159, 379.

[35] Rubin, V. C., Thonnard, N. and Ford, W. K. J., 1980, Astrophys. J., 238, 471.

[36] Sahni V., Saini T. D., Starobinsky A. A., Alam U., 2003, JETP Lett. 77, 201

[37] Salaris, M., Degl'innocenti, and Weiss, A., 1997, Astrophys. J., 479, 665.

[38] Shapiro, I. L. and Sola, J., 2000, Phys. Lett. B, 475, 236

[39] Sola, J., 2013, J. Phys. Conf. Ser., 453, 012015.

[40] Sola, J., Running Vacuum in the Universe: current phenomenological status, arXiv:1601.01668

[41] Sola, J., 2011, J. Phys.: Conf. Ser., 283, 012033.

[42] Sola, J., 2013, Phys. Conf. Ser., 453, 012015

[43] Steili, R., Boeckel, T. and Schaffner-Bielich, 2010, Phys.Rev. D, 8, 123513.

[44] Sukyong Yi et al., 2001, Astrophys. J. Suppl. S., 136, 417.

[45] Tyson, J. A., Kochanski, G. P. and Dell'Antonio, I. P., 1988, Astrophys. J., 498, L 107.

[46] Wilson J. R., Mathews, G. J., Fuller, G. M., Phys. 2007, Rev. D 75, 043521

[47] Zel'dovich, Ya. B., 1962, Sov.Phys. JETP, 14, 11437 . 
[48] Zel'dovich, Ya B., 1972, Mon. Not. R. Astron. Soc., 160, 1.

[49] Zimdahl, W., Schwarz, D. J., Balakin, A. B. and Pavn, D., 2001, Phys. Rev. D, 64, 063501.

[50] Zwicky, F., 1993, Helv. Phys. Acta., 6, 110. 